\title{
Two-dimensional concurrent HMQC-COSY as an approach for small molecule chemical shift assignment and compound identification
}

\author{
Kaifeng Hu • William M. Westler · John L. Markley
}

Received: 8 January 2011/ Accepted: 8 February 2011/Published online: 4 March 2011

(c) The Author(s) 2011. This article is published with open access at Springerlink.com

\begin{abstract}
Chemical shift assignment is the first step toward the structure elucidation of natural products and other chemical compounds. We propose here the use of 2D concurrent HMQC-COSY as an experiment for rapid chemical shift assignment of small molecules. This experiment provides well-dispersed ${ }^{1} \mathrm{H}-{ }^{13} \mathrm{C}$ peak patterns that are distinctive for different functional groups plus ${ }^{1} \mathrm{H}-{ }^{1} \mathrm{H}$ COSY connectivities that serve to identify adjacent groups. The COSY diagonal peaks, which are phased to be absorptive, resemble ${ }^{1} \mathrm{H}^{13} \mathrm{C}$ HMQC cross peaks. We demonstrate the applicability of this experiment for rapidly and unambiguously establishing correlations between different functional groups through the analysis of the spectrum of a metabolite (jasmonic acid) dissolved in $\mathrm{CDCl}_{3}$. In addition, we show that the experiment can be used to assign spectra of compounds in a mixture of metabolites in $\mathrm{D}_{2} \mathrm{O}$.
\end{abstract}

Keywords 2D HMQC-COSY - Chemical shift assignment $\cdot$ Compound identification .

Metabolite mixture

\section{Introduction}

2D COSY spectra (Aue et al. 1976) provide important information on the connectivity between different functional groups; however, 2D ${ }^{1} \mathrm{H}-{ }^{1} \mathrm{H}$ COSY spectra suffer from phasing issues, arising from intrinsic differences in the relative phase of diagonal and cross peaks, and peak

K. Hu · W. M. Westler · J. L. Markley $(\bowtie)$

National Magnetic Resonance Facility at Madison, University

of Wisconsin-Madison, Madison, WI 53706, USA

e-mail: markley@nmrfam.wisc.edu overlap issues, resulting from the low proton chemical shift dispersion. Heteronuclear 2D HSQC or HMQC experiments yield better-resolved spectra of metabolites and metabolite mixtures because of the higher chemical shift dispersion of carbon; however, these experiments do not offer information on the connectivity between different functional groups. Heteronuclear multiple-bond correlation spectra, HMBC type experiments (Bax and Summers 1986) can show heteronuclear long-range "indirect" connectivities, but these are not always unambiguous. The INADEQUATE experiment (Bax et al. 1980), which makes use of direct ${ }^{13} \mathrm{C}-{ }^{13} \mathrm{C}$ coupling, can provide very important information on ${ }^{13} \mathrm{C}-{ }^{13} \mathrm{C}$ connectivities within the molecular skeleton, but its application is limited by the low sensitivity of the experiment at natural abundance ${ }^{13} \mathrm{C}$.

To overcome the peak overlap issue in 2D COSY, the 3D ${ }^{13} \mathrm{C}$-edited HMQC-COSY experiment was proposed and demonstrated on kanamycin A with natural abundance ${ }^{13} \mathrm{C}$ (Fesik et al. 1989). A ${ }^{13} \mathrm{C}$-resolved COSY experiment involving selective excitation of a particular carbon signal by the SELINCOR technique was proposed as a means for overcoming spectral overlaps (Facke and Berger 1995). The 3D HMQC-COSY experiment was later modified with gradient-enhanced coherence order selection and further demonstrated on sucrose and menthol to facilitate assignments (Hurd and John 1991).

In 2D HMQC-COSY, the initial ${ }^{1} \mathrm{H}$ magnetization is transferred concurrently to ${ }^{13} \mathrm{C}$ through ${ }^{1} \mathrm{~J}_{\mathrm{CH}}$ (HMQC) and to ${ }^{1} \mathrm{H}$ through ${ }^{3} \mathrm{~J}_{\mathrm{HH}}$ (or ${ }^{\mathrm{n}} \mathrm{J}_{\mathrm{HH}}, \mathrm{COSY}$ ). The ${ }^{1} \mathrm{H}-{ }^{13} \mathrm{C}$ multiplequantum period is exploited for both ${ }^{13} \mathrm{C}$ chemical shift coding and for achieving a maximal COSY effect. Similar information on the connectivity can be obtained from HSQC-COSY to HSQC-TOCSY (Bax and Davis 1985) experiments. For HSQC-COSY and HSQC-TOCSY, the initial ${ }^{1} \mathrm{H}$ magnetization is transferred sequentially to 
bonded ${ }^{13} \mathrm{C}$ 's (by HSQC) and to homonuclear $J$ coupled ${ }^{1} \mathrm{H}$ 's (by COSY or TOCSY). Considering the relatively small 3-bond ${ }^{1} \mathrm{H}-{ }^{1} \mathrm{H}$ homonuclear $J$-couplings, usually the coupling Hamiltonian has to be allowed to evolve for a considerably long time to achieve reasonable COSY or TOCSY effects. In addition, to achieve reasonable resolution along the indirect ${ }^{13} \mathrm{C}$-dimension, magnetization on ${ }^{13} \mathrm{C}$ (single-quantum coherence) has to evolve for a long $t_{1}$ time.

Here we present 2D HMQC-COSY experiments that can be run as constant-time (Fig. 1a) or non-constant-time (Fig. 1b) versions and used for rapid spectral assignment of small molecules. In the constant-time version of 2D HMQCCOSY to achieve maximum COSY effect, given the relatively small 3 -bond ${ }^{1} \mathrm{H}-{ }^{1} \mathrm{H}$ homonuclear $J$-couplings, the coupling Hamiltonian evolves during both the ${ }^{1} \mathrm{H}-{ }^{13} \mathrm{C}$ heteronuclear multiple quantum generation period $(\mathrm{a}-\mathrm{b})$ and the ${ }^{1} \mathrm{H}-{ }^{13} \mathrm{C}$ multiple quantum constant-time period. In the nonconstant-time version of 2D HMQC-COSY, the ${ }^{1} \mathrm{H}-{ }^{1} \mathrm{H}$ homonuclear $J$-coupling runs in accordion mode (Mandel and Palmer 1994). The constant-time version is superior for the analysis of small molecule spectra because of its higher COSY transfer efficiency with small $J$-couplings and its higher resolution along the indirect ${ }^{13} \mathrm{C}$-dimension compared to the non-constant-time version.

We illustrate the utility of this approach for the spectral assignment of a small molecule and show how the experiment can be used for identifying compounds in mixtures of metabolites.

\section{Materials and methods}

Jasmonic acid (Sigma-Aldrich) was dissolved in $\mathrm{CDCl}_{3}$ at a concentration about $60 \mathrm{mM}$. The constant-time 2D HMQC-COSY spectrum of jasmonic acid was recorded at $25^{\circ} \mathrm{C}$ on a Bruker Avance $500 \mathrm{MHz}$ spectrometer equipped with a $z$-gradient triple resonance CPTXO probe optimized for ${ }^{13} \mathrm{C}$ detection. The radio-frequency pulses on ${ }^{1} \mathrm{H}$ and ${ }^{13} \mathrm{C}$ were applied at 4.7 and $73 \mathrm{ppm}$, respectively. ${ }^{13} \mathrm{C}$ decoupling was carried out using GARP with a field strength of $\gamma B_{2}=3.38 \mathrm{kHz} .1024 \times 150$ complex data points were collected along ${ }^{1} \mathrm{H}$ and ${ }^{13} \mathrm{C}$ dimensions (spectral widths of 14 and $150 \mathrm{ppm}$, respectively) with 4 scans per FID and an inter-scan delay of $3 \mathrm{~s}$, resulting in a total data acquisition time of about $1 \mathrm{~h}$. A mixture containing $63.8 \mathrm{mM}$ alanine, $88.47 \mathrm{mM}$ methionine, and $124.36 \mathrm{mM}$ sodium 3-hydroxybutyrate (Sigma-Aldrich) was prepared in $\mathrm{D}_{2} \mathrm{O}$. The constant-time $2 \mathrm{D}$ HMQC-COSY spectrum of this mixture was collected at $25^{\circ} \mathrm{C}$ on a Bruker DMX $500 \mathrm{MHz}$ spectrometer equipped with a $z$-gradient triple resonance TCI probe. The radio-frequency pulses on ${ }^{1} \mathrm{H}$ and ${ }^{13} \mathrm{C}$ were applied at 4.7 and $43 \mathrm{ppm}$, respectively.
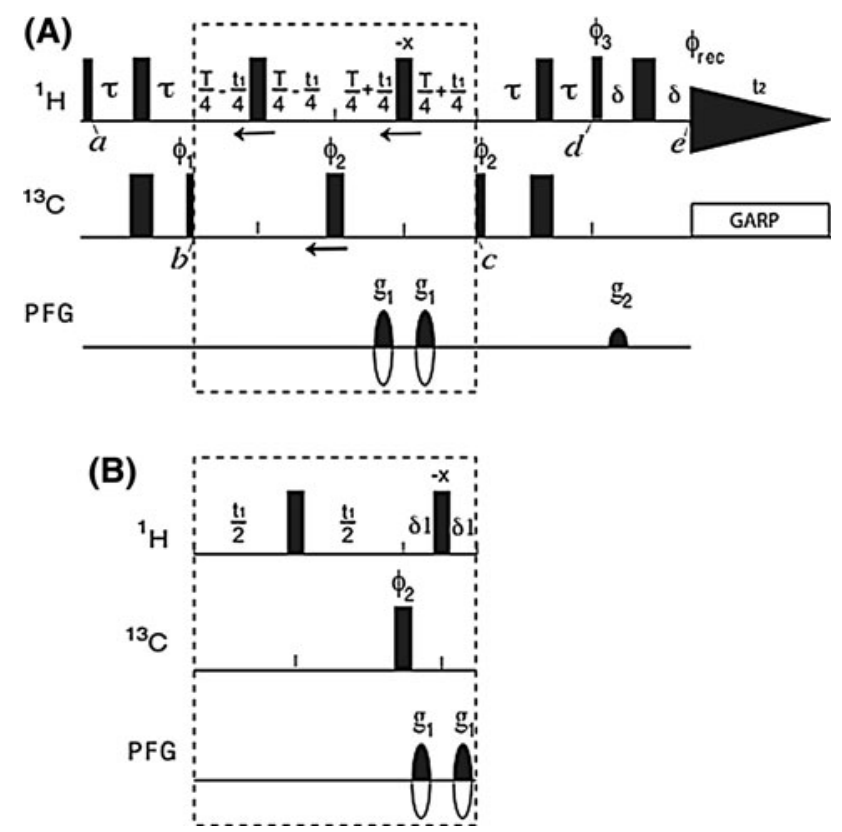

Fig. 1 a Constant-time 2D HMQC-COSY pulse sequence. Narrow and wide black bars indicate $90^{\circ}$ and $180^{\circ}$ pulses, respectively. The delays are $\tau=1.8 \mathrm{~ms} ; T=20 \mathrm{~ms}$ and $\delta=1.21 \mathrm{~ms}$. The phase cycling is as follows: $\phi_{1}=\mathrm{x},-\mathrm{x} ; \phi_{2}=\mathrm{x}, \mathrm{x}, \mathrm{x}, \mathrm{x},-\mathrm{x},-\mathrm{x},-\mathrm{x},-\mathrm{x}$; $\phi_{3}=\mathrm{y}, \mathrm{y},-\mathrm{y},-\mathrm{y} ; \phi_{\mathrm{rec}}=\mathrm{x},-\mathrm{x}, \mathrm{x},-\mathrm{x},-\mathrm{x}, \mathrm{x},-\mathrm{x}, \mathrm{x}$. All other radio-frequency pulses are applied with phase $\mathrm{x}$ except as indicated. Quadrature detection in the ${ }^{13} \mathrm{C}\left(t_{1}\right)$ dimension is achieved by using echo-antiecho-TPPI applied to the phase $\phi_{1}$ and by flipping the polarity of both gradients $g_{1}$ on every another FID (as indicated by the filled and open sine bells). The duration and strength of the coherence selective pulsed field gradients applied along the $z$-axis are: $g_{1}: 1 \mathrm{~ms}$, $26.5 \mathrm{G} / \mathrm{cm}$ and $g_{2}: 1 \mathrm{~ms}, 13.33 \mathrm{G} / \mathrm{cm}$ followed by a gradient recovery period of $200 \mu \mathrm{s}$. b Non-constant-time 2D HMQC-COSY pulse sequence. The constant-time period (dashed block in panel a) is replaced by a non-constant-time block. The short delay $\delta_{1}=1.21 \mathrm{~ms}$ is just to accommodate the coherence selective pulsed field gradients $g_{1}$, including the gradient recovery period of $200 \mu \mathrm{s}$

${ }^{13} \mathrm{C}$ GARP decoupling was carried out with a field strength of $\gamma B_{2}=3.38 \mathrm{kHz} .1024 \times 120$ complex data points were recorded along the ${ }^{1} \mathrm{H}$ and ${ }^{13} \mathrm{C}$ dimensions (spectral widths of 14 and 80 ppm, respectively) with 4 scans per FID and an inter-scan delay of $4 \mathrm{~s}$, resulting in total data acquisition time of about $1 \mathrm{~h}$. The NMRPipe package (Delaglio et al. 1995) was used to process spectra with linear prediction and zero-filling along the ${ }^{13} \mathrm{C}$ dimension.

\section{Results and discussion}

NMR pulse sequences

In the constant-time 2D concurrent HMQC-COSY pulse scheme (Fig. 1a), the initial ${ }^{1} \mathrm{H}^{a}$ polarization is excited by a $90^{\circ}$ pulse on ${ }^{1} \mathrm{H}$ followed by a multiple quantum generation period, where magnetization is transferred to ${ }^{1} \mathrm{H}^{a}-{ }^{13} \mathrm{C}^{a}$ multiple quantum coherence at point $b$. By synchronously 
shifting the $180^{\circ}{ }^{13} \mathrm{C}$ pulse $\left(\phi_{2}\right)$ and $180^{\circ}{ }^{1} \mathrm{H}$ pulses (as indicated by the arrows) during the constant time period $T$ (points $b-c$ shown in the dashed rectangle), the chemical shift of ${ }^{13} \mathrm{C}^{a}$ is encoded in time and concurrently the ${ }^{1} \mathrm{H}-{ }^{1} \mathrm{H}$ homonuclear $J$-coupling Hamiltonian evolves. Note, that besides refocusing the chemical shift evolution of ${ }^{1} \mathrm{H}^{a}$, insertion of the two $180^{\circ}{ }^{1} \mathrm{H}$ pulses during the constant time also refocuses the evolution of other passive heteronuclear $J$-couplings between ${ }^{13} \mathrm{C}^{a}$ and other multiple bond coupled protons. Thus, a narrower line width along the ${ }^{13} \mathrm{C}$ dimension can be obtained. Application of a second $90^{\circ}$ pulse on ${ }^{13} \mathrm{C}\left(\phi_{2}\right)$ transfers ${ }^{1} \mathrm{H}^{a}-{ }^{13} \mathrm{C}^{a}$ MQ coherence back to ${ }^{1} \mathrm{H}^{a}$. Following the $2 \tau$ reverse HMQC period, magnetization on ${ }^{1} \mathrm{H}^{a}$ evolves in-phase relative to ${ }^{13} \mathrm{C}^{a}$. For the entire $2 \tau+T+2 \tau$ time period (point $a-d$ ), ${ }^{1} \mathrm{H}-{ }^{1} \mathrm{H}$ homonuclear $J$-coupling Hamiltonian evolves and part of the magnetization arising from ${ }^{1} \mathrm{H}^{a}$ evolves to be antiphase relative to the homonuclear coupled third party proton ${ }^{1} \mathrm{H}^{b}$. COSY transfer from ${ }^{1} \mathrm{H}^{a}$ to ${ }^{1} \mathrm{H}^{b}$ is then achieved by the $90^{\circ}$ pulse on ${ }^{1} \mathrm{H}\left(\phi_{3}\right)$. The period $2 \delta$ inserted before detection serves to accommodate the refocusing gradient $g_{2}$. The relevant coherence transfer pathway can be described as:

$$
\begin{aligned}
& H_{z}^{a} \stackrel{90_{X}^{\circ}}{\longrightarrow}-H_{y}^{a}(a) \stackrel{180_{H}^{\circ}, 180_{C}^{\circ}, 2 \tau}{\longrightarrow} 2 H_{x}^{a} C_{z}^{a} \cos \left[\pi J_{H^{a} H^{b}} \cdot 2 \tau\right] \\
& +4 H_{y}^{a} H_{z}^{b} C_{z}^{a} \sin \left[\pi J_{H^{a} H^{b}} \cdot 2 \tau\right] \\
& \stackrel{90_{\phi 1}^{\circ}}{\longrightarrow} 2 H_{x}^{a} C_{-y}^{a} \cos \left[\pi J_{H^{a} H^{b}} \cdot 2 \tau\right] \\
& +4 H_{y}^{a} H_{z}^{b} C_{-y}^{a} \sin \left[\pi J_{H^{a} H^{b}} \cdot 2 \tau\right](b) \\
& \stackrel{T, g_{1}}{\longrightarrow} 2 H_{x}^{a} C_{-y}^{a}\left(\omega_{C^{a}}, \gamma_{C} g_{1}\right) \cos \left[\pi J_{H^{a} H^{b}} \cdot(T+2 \tau)\right] \\
& +4 H_{y}^{a} H_{z}^{b} C_{-y}^{a}\left(\omega_{C^{a}}, \gamma_{C} g_{1}\right) \sin \left[\pi J_{H^{a} H^{b}} \cdot(T+2 \tau)\right](c) \\
& \stackrel{90_{\phi 2}^{\circ}}{\longrightarrow} 2 H_{x}^{a} C_{z}^{a}\left(\omega_{C^{a}}, \gamma_{C} g_{1}\right) \cos \left[\pi J_{H^{a} H^{b}} \cdot(T+2 \tau)\right] \\
& +4 H_{y}^{a} H_{z}^{b} C_{z}^{a}\left(\omega_{C^{a}}, \gamma_{C} g_{1}\right) \sin \left[\pi J_{H^{a}} H^{b} \cdot(T+2 \tau)\right] \\
& \stackrel{180_{H}^{\circ}, 180_{C}^{\circ}, 2 \tau}{\longrightarrow} H_{y}^{a}\left(\omega_{C^{a}}, \gamma_{C} g_{1}\right) \cos \left[\pi J_{H^{a}} H^{b} \cdot(T+4 \tau)\right] \\
& +2 H_{-x}^{a} H_{z}^{b}\left(\omega_{C^{a}}, \gamma_{C} g_{1}\right) \sin \left[\pi J_{H^{a} H^{b}} \cdot(T+4 \tau)\right](d) \\
& \stackrel{90_{\phi 3}^{\circ}}{\longrightarrow} H_{y}^{a}\left(\omega_{C^{a}}, \gamma_{C} g_{1}\right) \cos \left[\pi J_{H^{a}} H^{b} \cdot(T+4 \tau)\right] \\
& +2 H_{z}^{a} H_{x}^{b}\left(\omega_{C^{a}}, \gamma_{C} g_{1}\right) \sin \left[\pi J_{H^{a}} H^{b} \cdot(T+4 \tau)\right] \\
& \stackrel{180_{H}^{\circ}, 2 \delta, g_{2}}{\longrightarrow} H_{y}^{a}\left(\omega_{C^{a}}\right) \cos \left[\pi J_{H^{a} H^{b}} \cdot(T+4 \tau)\right] \\
& +2 H_{z}^{a} H_{x}^{b}\left(\omega_{C^{a}}\right) \sin \left[\pi J_{H^{a}} H^{b} \cdot(T+4 \tau)\right](e)
\end{aligned}
$$

The term $\left(\omega_{C^{a}}, \gamma_{C} g_{1}\right)$ arises from the coherence encoded by the chemical shift of ${ }^{13} \mathrm{C}^{a}\left(\omega_{C^{a}}\right)$ and dephased by gradient $g_{1}$. Gradient selection is then achieved by application of the refocusing gradient $g_{2}$ [last line in Eq. (1)]. Note that the homonuclear $J$-coupling evolution is ignored during the short gradient refocusing period $2 \delta$.

The COSY diagonal peak, represented by the first term at point $e, H_{y}^{a}\left(\omega_{C^{a}}\right) \cos \left[\pi J_{H^{a} H^{b}} \cdot(T+4 \tau)\right]$, is $90^{\circ}$ out of phase relative to the COSY cross peak, represented by the second term at point $e, 2 H_{z}^{a} H_{x}^{b}\left(\omega_{C^{a}}\right) \sin \left[\pi J_{H^{a} H^{b}} \cdot(T+4 \tau)\right]$. Therefore, whereas the COSY diagonal peaks are phased to be absorptive, the COSY cross peaks show a typical pattern of antiphase dispersion (see the line shape of $\mathrm{H} 12$ in the dashed rectangle in Fig. 2b). The COSY diagonal peak term $H_{y}^{a}\left(\omega_{C^{a}}\right) \cos \left[\pi J_{H^{a} H^{b}} \cdot(T+4 \tau)\right]$ evolves under the chemical shift of $\mathrm{H}^{a}$ during detection, which will appear as normal ${ }^{1} \mathrm{H}^{a}-{ }^{13} \mathrm{C}^{a} \mathrm{HMQC}$ cross-peaks. The COSY cross peak term $2 H_{z}^{a} H_{x}^{b}\left(\omega_{C^{a}}\right) \sin \left[\pi J_{H^{a} H^{b}} \cdot(T+4 \tau)\right]$, will evolve under the chemical shift of $\mathrm{H}^{b}$ during detection. It will thus show cross peaks at the chemical shift of ${ }^{13} \mathrm{C}^{a}$ and chemical shift of the coupled proton $\mathrm{H}^{b}$. This indirect correlation provides information similar to that of an HMBC experiment. Similarly, magnetization initially from ${ }^{1} \mathrm{H}^{b}$, will yield a COSY diagonal peak at ${ }^{1} \mathrm{H}^{b}-{ }^{13} \mathrm{C}^{b}$ and a COSY cross peak at ${ }^{1} \mathrm{H}^{a}-{ }^{13} \mathrm{C}^{b}$.

Therefore, in the 2D HMQC-COSY spectrum, neighboring $\left({ }^{1} \mathrm{H}-{ }^{1} \mathrm{H}\right.$ homonuclear coupled) functional groups ${ }^{1} \mathrm{H}^{a}-{ }^{13} \mathrm{C}^{a}$ and ${ }^{1} \mathrm{H}^{b}-{ }^{13} \mathrm{C}^{b}$ will yield two COSY diagonal peaks at the chemical shifts of ${ }^{13} \mathrm{C}^{a} /{ }^{1} \mathrm{H}^{a}$ and ${ }^{13} \mathrm{C}^{b} /{ }^{1} \mathrm{H}^{b}$, respectively, which can be correlated by the two COSY cross peaks at the chemical shifts of ${ }^{13} \mathrm{C}^{a} /{ }^{1} \mathrm{H}^{b}$ and ${ }^{13} \mathrm{C}^{b} /{ }^{1} \mathrm{H}^{a}$. The COSY diagonal and cross peaks can be recognized by their line shape: the COSY diagonal peaks are phased to be absorptive, and the COSY cross peaks show an antiphase dispersive line shape. They also can be differentiated simply by comparison to conventional HSQC/HMQC spectra: the extra peaks shown in HMQC-COSY are the COSY cross peaks, giving HMBC type information. The feature of high chemical shift dispersion along the ${ }^{13} \mathrm{C}$ dimension and HMBC type information contained in the HMQC-COSY spectrum renders the HMQC-COSY experiment a robust approach for chemical shift assignments and also for identifying compounds in metabolite mixtures as demonstrated below.

In the non-constant-time version of HMQC-COSY, the constant-time period (dashed block in Fig. 1a) is replaced by non-constant-time block (Fig. 1b). The short delay $\delta_{1}=1.21 \mathrm{~ms}$ is just to accommodate the coherence selective pulsed field gradients $g_{1}$. As the chemical shift of ${ }^{13} \mathrm{C}$ is encoded in time, the effective evolution time for the ${ }^{1} \mathrm{H}-{ }^{1} \mathrm{H}$ homonuclear $J$-coupling Hamiltonian also varies with $t_{1}$, from $\left(2 \tau+4 \delta_{1}+2 \tau\right)$ to $\left(2 \tau+t_{1}+2 \delta_{1}+2 \tau\right)$ (point $a-d$ ). ${ }^{1} \mathrm{H}-{ }^{1} \mathrm{H}$ homonuclear $J$-coupling evolution in the accordion mode allows the COSY transfer in the nonconstant-time 2D HMQC-COSY to be more efficient for very diverse values of $J_{\mathrm{HH}}$.

\section{Chemical shift assignment of jasmonic acid}

We illustrate the application of this approach with data from jasmonic acid (see Fig. 2a for the structure and atom 


\section{(A) HMQC-COSY}

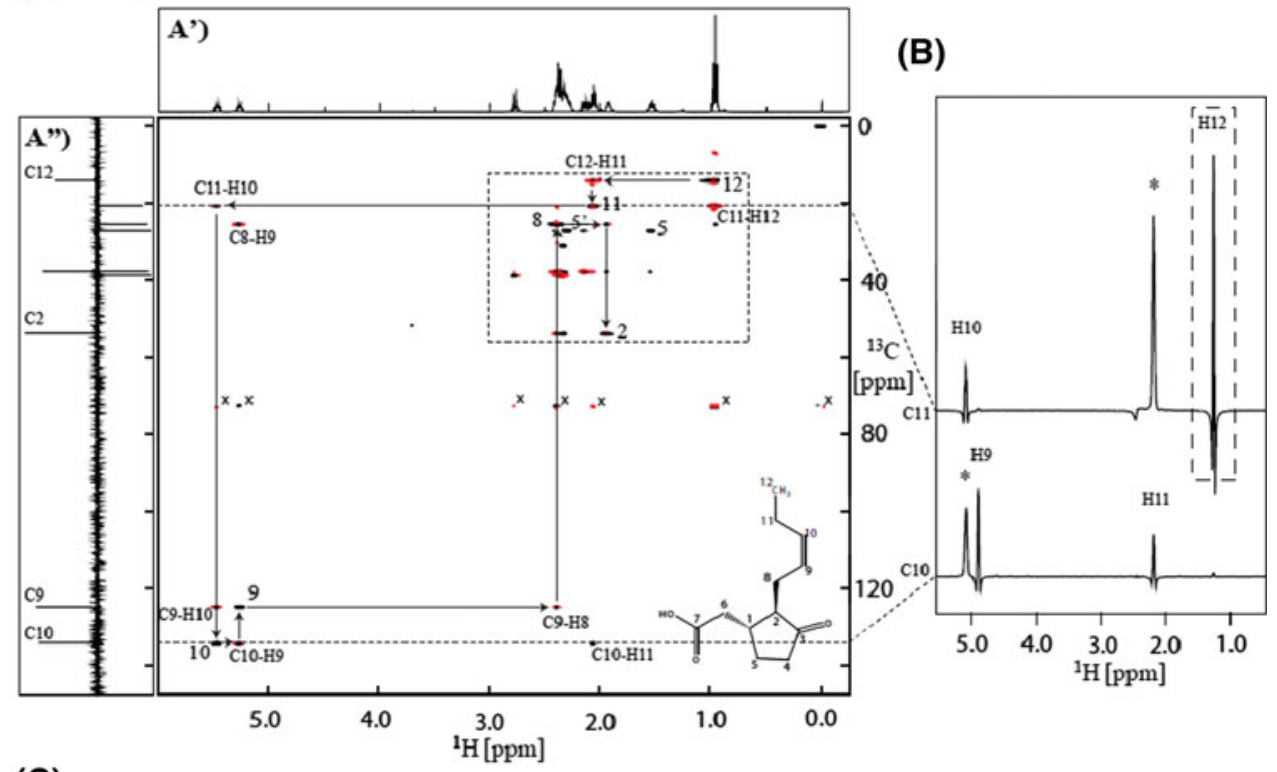

(C)

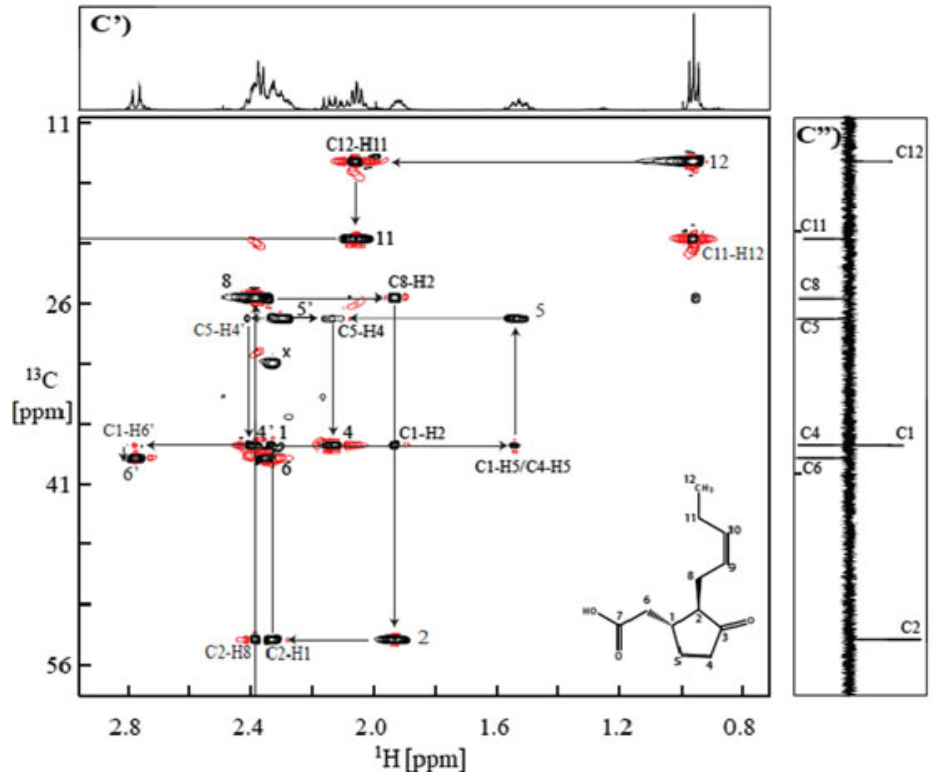

Fig. 2 a $500 \mathrm{MHz}$ constant time 2D HMQC-COSY spectrum of jasmonic acid in $\mathrm{CDCl}_{3}$. The structure and atom nomenclature of jasmonic acid are shown at bottom-right. The COSY diagonal peaks are labeled with atom numbers, while the COSY cross peaks are labeled to identify the carbon and correlated proton. The arrows starting from 12 illustrate the stepwise path for the chemical shift assignment (see the text for details.). Panels $a^{\prime}$ and $a^{\prime \prime}$, respectively, show for reference $1 \mathrm{D}{ }^{1} \mathrm{H}$ and ${ }^{13} \mathrm{C}$ DEPT-135 spectra of jasmonic acid downloaded from BMRB (http://www.bmrb.wisc.edu/). Axial artifacts are marked with " $x$ "; the axial peaks are shifted by TPPI quadrature detection and correspond to peaks in the ${ }^{1} \mathrm{H}$ dimension; thus, we attribute them to ${ }^{12} \mathrm{C}$ species (98.9\% abundance). b $1 \mathrm{D}$ slices along the ${ }^{1} \mathrm{H}$ dimension taken from the 2D HMQC-COSY spectrum at

nomenclature of this compound as well as its 2D HMQCCOSY). The signal from the methyl group (C12-H12, labelled peak 12) of jasmonic acid is easily identified on the the chemical shift of $\mathrm{C} 11$ and $\mathrm{C} 10$. The COSY diagonal peaks are marked with asterisks *; the COSY cross peaks are labeled with the correlated protons. While the COSY diagonal peaks are phased to be absorptive, the COSY cross peaks show a typical pattern of antiphase dispersion, as shown in the broken box. The excellent alignment of the chemical shifts of $\mathrm{H} 10$ and $\mathrm{H} 11$ from these two slices indicates the connectivity between $\mathrm{C} 11$ and $\mathrm{C} 10$. $\mathbf{c}$ Expansion of the crowed region boxed by a dashed line in a. The detailed pathway for chemical shift assignments is indicated by the arrows. Panels $c^{\prime}$ and $c^{\prime \prime}$ show the expanded $1 \mathrm{D}{ }^{1} \mathrm{H}$ and ${ }^{13} \mathrm{C}$ DEPT-135 spectra of jasmonic acid. The geminal protons attached to $\mathrm{C} 4, \mathrm{C} 5$ and $\mathrm{C} 6$, which have different chemical shifts, are labeled as $\mathrm{H}$ and $\mathrm{H}^{\prime}$

basis of its characteristic ${ }^{1} \mathrm{H}$ and ${ }^{13} \mathrm{C}$ chemical shifts. The assignment of the methyl group can be confirmed by reference to $1 \mathrm{D}{ }^{1} \mathrm{H}$ (Fig. $2 \mathrm{a}^{\prime}$ ) and ${ }^{13} \mathrm{C}$ DEPT-135 (Fig. 2a") 


\section{(A) HMQC-COSY}

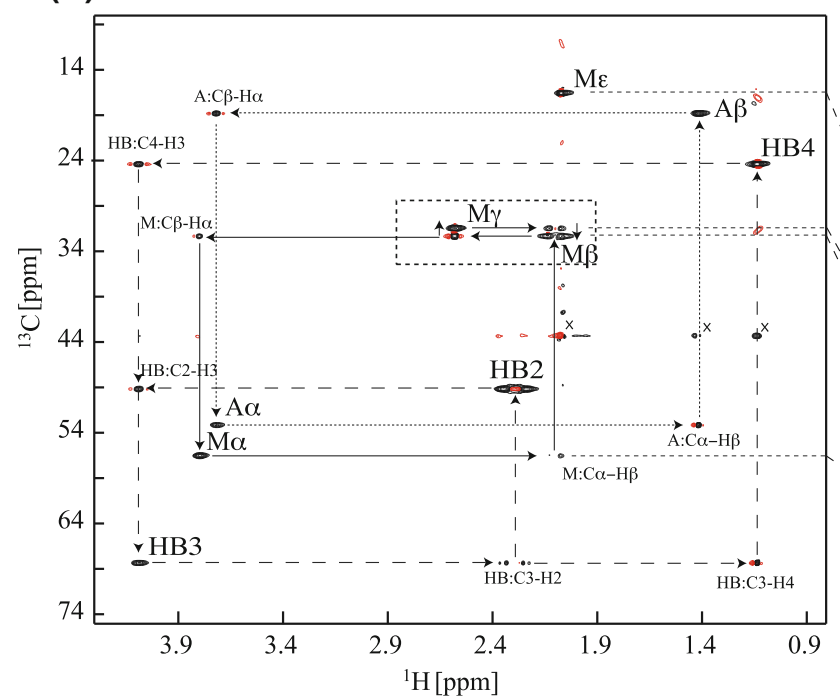

(B)

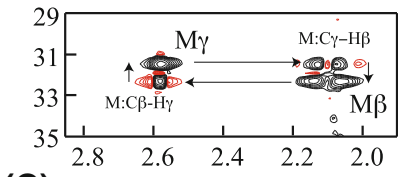

(C)

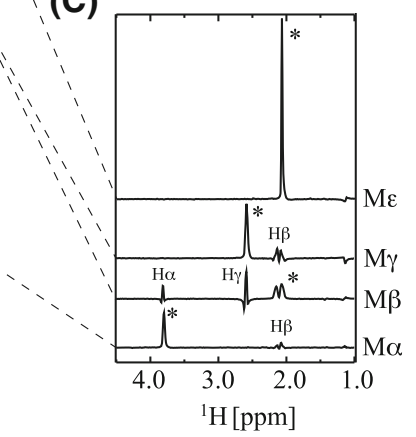<smiles>CC(O)CC(=O)[O-]</smiles>

3-Hydroxybutyrate (HB)

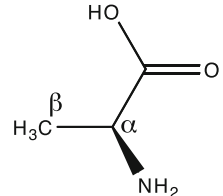

L-Alanine (A)

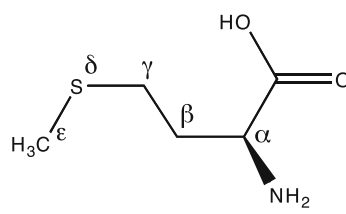

L-Methionine (M)
Fig. 3 a $500 \mathrm{MHz}$ constant-time 2D HMQC-COSY spectrum of the mixture of alanine (A), methionine (M), and sodium 3-hydroxybutyrate $(\mathrm{HB})$ in $\mathrm{D}_{2} \mathrm{O}$. The structures and atom nomenclature for these compounds are shown at the bottom of the figure. The COSY diagonal peaks are labeled with the abbreviated name of the molecule and atom designator. The COSY cross peaks are labeled with the abbreviated name of the molecule and the atom designators of the carbon and its correlated long-range proton. The spin networks of $\mathrm{A}, \mathrm{M}$, and $\mathrm{HB}$ are identified by the dotted, solid, and broken arrows. Axial artifacts are marked with " $x$ "; their origin is as described in the caption to Fig. 2. b Expansion of the region enclosed by the dashed rectangle in

spectra. Walking along the ${ }^{1} \mathrm{H}$ dimension to the left of peak 12 at the ${ }^{13} \mathrm{C}$ chemical shift of the methyl group (14.21 ppm) leads to a cross peak at ${ }^{1} \mathrm{H}=2.058 \mathrm{ppm}$, which corresponds to the indirect interaction of $\mathrm{C} 12$ and $\mathrm{H} 11(\mathrm{C} 12-\mathrm{H} 11)$. Then walking down from peak $\mathrm{C} 12-\mathrm{H} 11$ along the ${ }^{13} \mathrm{C}$ dimension leads to a cross peak at ${ }^{13} \mathrm{C}=20.80 \mathrm{ppm}$ (peak 11, the direct H11-C11 correlation). Next, walking leftward from peak 11 along the ${ }^{1} \mathrm{H}$ dimension leads to a cross peak at ${ }^{1} \mathrm{H}=5.463 \mathrm{ppm}$, which corresponds to $\mathrm{C} 11-\mathrm{H} 10$. Again, the downward arrow from peak $\mathrm{C} 11-\mathrm{H} 10$ along the ${ }^{13} \mathrm{C}$ dimension leads to the cross peak at ${ }^{13} \mathrm{C}=134.35 \mathrm{ppm}$, from the direct $\mathrm{C} 10-\mathrm{H} 10$ correlation (peak 10). This assigns C10 (134.35 ppm) and H10 (5.463 ppm). As noted above, the direct and indirect correlation peaks can be distinguished by their line shapes; these are illustrated by $1 \mathrm{D}$ slices along the ${ }^{1} \mathrm{H}$ dimension taken from the 2D HMQC-COSY spectrum at the chemical shift of C11 (20.80 ppm) and C10 (134.35 ppm) (Fig. 2b). The directly correlated peaks a shows the neighboring connectivity between $\mathrm{M} \mathrm{C}^{\beta}$ and $\mathrm{C}^{\gamma}$. c Shown as an example are 1D slices along the ${ }^{1} \mathrm{H}$ dimension taken from the 2D HMQC-COSY spectrum at the chemical shifts of $\mathrm{M} \mathrm{C}^{\varepsilon}, \mathrm{C}^{\gamma}, \mathrm{C}^{\beta}$ and $\mathrm{C}^{\alpha}$. The COSY diagonal peaks are marked with asterisks *; the COSY cross peaks are labeled with the correlated protons. The excellent alignment of the chemical shifts of $\mathrm{H}^{\gamma}, \mathrm{H}^{\beta}$ and $\mathrm{H}^{\alpha}$ from these slices and the correlation of $\mathrm{C}^{\beta}$ with both $\mathrm{H}^{\gamma}$ and $\mathrm{H}^{\alpha}$ indicate that the spin network is connected as $C^{\gamma}-C^{\beta}-C^{\alpha}$. The slice at Met $C^{\varepsilon}$ does not show any COSY cross peak except for the COSY diagonal peak marked by an asterisks. $\mathrm{C}^{\varepsilon}$ is thus isolated from the rest of the spin network

(COSY diagonal peaks, marked with asterisks *) are phased to be absorptive, and the indirectly correlated (long range) peaks (COSY cross peaks, labeled with the correlated protons) show an antiphase dispersive lineshape (see the inset showing H12). The excellent alignment of the chemical shifts of $\mathrm{H} 10$ and $\mathrm{H} 11$ from these two slices indicates the neighboring connectivity between $\mathrm{C} 11$ and $\mathrm{C} 10$. Figure $2 \mathrm{c}$ shows an expansion of the crowed region outlined by the dashed line in Fig. 2a. By following the arrows in Fig. 2a and $\mathrm{c}$ and by repeating the above procedure, the ${ }^{13} \mathrm{C}$ and ${ }^{1} \mathrm{H}$ chemical shift assignments can be extended from to the rest of the molecule. Carbons C4, C5, and C6 each have two attached protons, designated by unprimed and primed labels (e.g., $\mathrm{H} 4$ and $\mathrm{H} 4^{\prime}$ ). Due to the relatively low resolution along the indirect ${ }^{13} \mathrm{C}$ dimension of 2D HMQC-COSY, C4 and C1 are assigned to the same chemical shift. These assignments can be easily confirmed by comparison to the ${ }^{13} \mathrm{C}$ DEPT- 135 spectrum (panel $2 \mathrm{c}^{\prime \prime}$ ), in which $\mathrm{C} 1$ is positive and $\mathrm{C} 4$ is 
negative. Only the quaternary carbons of jasmonic acid (C3 and $\mathrm{C} 7$ ) remain undetected and unassigned by the $2 \mathrm{D}$ HMQC-COSY approach. They can be found by $1 \mathrm{D}{ }^{13} \mathrm{C}$ NMR detection.

\section{Compound identification from metabolite mixture}

The 2D HMQC-COSY experiment can also be used to identify individual compounds in a mixture of metabolites. We illustrate this by reference to results with a mixture of $63.75 \mathrm{mM}$ alanine (A), $88.47 \mathrm{mM}$ methionine (M), and $124.36 \mathrm{mM}$ sodium 3-hydroxybutyrate $(\mathrm{HB})$ in $\mathrm{D}_{2} \mathrm{O}$. Figure $3 \mathrm{a}$ shows the 2D HMQC-COSY spectrum of the mixture. As before, the directly correlated peaks are absorptive while the indirectly correlated peaks have an antiphase dispersive lineshape. Following the procedure outlined above for jasmonic acid, the spin networks of A, $\mathrm{M}$, and HB were identified, as illustrated by the dotted, solid, and broken arrows, respectively, in Fig. 3a and b. Slices from the 2D HMQC-COSY spectrum of the mixture taken along the ${ }^{1} \mathrm{H}$ dimension at the chemical shifts of $\mathrm{M}$ $\mathrm{C}^{\varepsilon}, \mathrm{C}^{\gamma}, \mathrm{C}^{\beta}$ and $\mathrm{C}^{\alpha}$ (Fig. 3c) show how this spin system can be identified selectively; the excellent alignment of the chemical shifts of $\mathrm{H}^{\gamma}, \mathrm{H}^{\beta}$ and $\mathrm{H}^{\alpha}$ from these slices indicates that these groups belong to the same spin network. Because $\mathrm{C}^{\beta}$ shows indirect correlation with both $\mathrm{H}^{\gamma}$ and $\mathrm{H}^{\alpha}$, whereas $\mathrm{C}^{\gamma}$ and $\mathrm{C}^{\alpha}$, each show indirect correlations only with $\mathrm{H}^{\beta}$, the spin network is connected as $\mathrm{C}^{\gamma}-\mathrm{C}^{\beta}-\mathrm{C}^{\alpha}$. The slice at $\mathrm{M}$ $\mathrm{C}^{\varepsilon}$ does not show any indirectly correlated peak because its attached proton is not coupled to any other group by efficient COSY transfer; thus, $\mathrm{C}^{\varepsilon}$ is isolated from the rest of the spin network. Having identified the spin network and associated chemical shifts, the identity of the compound can be determined from a metabolite standards database.

\section{Conclusion}

The 2D HMQC-COSY experiment presented here offers a robust approach for assigning ${ }^{1} \mathrm{H}$ and ${ }^{13} \mathrm{C}$ NMR signals from individual compounds or mixtures of compounds. Because of the high chemical shift dispersion along the carbon dimension, this experiment produces distinct peak patterns for different functional groups through ${ }^{1} \mathrm{H}-{ }^{13} \mathrm{C}$ multiple quantum coherence while retaining the important information on peak connectivity through ${ }^{1} \mathrm{H}-{ }^{1} \mathrm{H}$ COSY. We have implemented this method as a routine experiment for chemical shift assignment of standard compounds being added to the BMRB metabolomics database (http://www. bmrb.wisc.edu/metabolomics) in conjunction with $2 \mathrm{D}$
${ }^{1} \mathrm{H}-{ }^{13} \mathrm{C}$ HMBC for assignment of quaternary carbons. Although the spectra shown here were of compounds at high concentration $(60 \mathrm{mM})$, we have used the approach with samples at $2 \mathrm{mM}$ concentration with only 4 transients. With more extensive time averaging, we expect that $2 \mathrm{D}$ HMQC-COSY can be used for the assignment of compounds at concentrations as low as $500 \mu \mathrm{M}$. We anticipate that the experiment may find wider application in NMR studies of metabolites and natural products.

Acknowledgments The authors thank James J. Ellinger for preparing the metabolite mixture and Dr. Ravi Rapolu for the sample of jasmonic acid. This work was supported by NIH grant RR02301 and by the DOE Great Lakes Bioenergy Research Center (DOE BER Office of Science DE-FC02-07ER64494). NMR data were acquired at the National Magnetic Resonance Facility at Madison.

Open Access This article is distributed under the terms of the Creative Commons Attribution Noncommercial License which permits any noncommercial use, distribution, and reproduction in any medium, provided the original author(s) and source are credited.

\section{References}

Aue WP, Bartholdi E, Ernst RR (1976) 2-Dimensional spectroscopy-application to nuclear magnetic-resonance. J Chem Phys 64:2229-2246

Bax A, Davis DG (1985) Homonuclear Hartmann-Hahn magnetization transfer: new one- and two-dimensional NMR methods for structure determination and spectral assignment MLEV-17 based two-dimensional homonuclear magnetization transfer spectroscopy. J Magn Reson 65:355-360

Bax A, Summers MF (1986) H-1 and C-13 assignments from sensitivity-enhanced detection of heteronuclear multiple-bond connectivity by $2 \mathrm{D}$ multiple quantum NMR. J Am Chem Soc 108:2093-2094

Bax A, Freeman R, Kempsell SP (1980) Natural abundance carbon13-carbon-13 coupling observed via double-quantum coherence. J Am Chem Soc 102:4849-4851

Delaglio F, Grzesiek S, Vuister GW, Zhu G, Pfeifer J, Bax A (1995) NMRPIPE - a multidimensional spectral processing system based on UNIX pipes. J Biomol NMR 6:277-293

Facke T, Berger S (1995) Gradient-enhanced selincor for selective excitation in a C-13-resolved cosy experiment. Magn Reson Chem 33:144-148

Fesik SW, Gampe RT, Zuiderweg ERP (1989) Heteronuclear 3-Dimensional NMR-spectroscopy-natural abundance c-13 chemical-shift editing of $\mathrm{H}-1-\mathrm{H}-1$ cosy spectra. J Am Chem Soc 111:770-772

Hurd RE, John BK (1991) 3-Dimensional gradient-enhanced relayedited proton spectroscopy, GREP-HMQC-COSY. J Magn Reson 92:658-668

Mandel AM, Palmer AG (1994) Measurement of relaxation-rate constants using constant-time accordion NMR-spectroscopy. J Magn Reson A 110:62-72 